\title{
Extracting atoms from molecular electron densities via integral equations
}

\author{
Andrew T. B. Gilbert ${ }^{\mathrm{a})}$ and Peter M. W. Gill \\ School of Chemistry, University of Nottingham, Nottingham NG7 2RD, United Kingdom \\ Stephen W. Taylor \\ Department of Mathematics, University of Auckland, Auckland, New Zealand
}

(Received 11 December 2003; accepted 10 February 2004)

\begin{abstract}
The observation that a molecular electron density is close to the superposition of its constituent atoms leads naturally to the idea of modeling a density by a sum of nuclear-centered, spherically symmetric functions. The functions that are optimal in a least-squares sense are known as Stewart atoms. Previous attempts to construct Stewart atoms by expanding them in an auxiliary basis have been thwarted by slow convergence with respect to the size of the auxiliary basis used. We present a method for constructing Stewart atoms via convolution integrals which bypasses the need for an auxiliary basis, and is able to produce highly accurate approximations to Stewart atoms. (c) 2004 American Institute of Physics. [DOI: 10.1063/1.1691401]
\end{abstract}

\section{INTRODUCTION}

The modeling of chemical phenomenon in silico has become increasingly important. This is due, in part, to the escalation of computing power, but also to the development of models that are simple enough to apply to large molecules, yet are still able preserve the essential properties of the system. Density-functional theory made a significant contribution by legitimizing the focus on the charge density, $\rho(\mathbf{r})$, of a system rather than the more recondite wave function. However, even $\rho(\mathbf{r})$ is a complicated function in 3-space, and it is worth considering if further simplification is possible. Stewart's suggestion ${ }^{1,2}$ was to model the density by spherical, nuclear-centered functions. These functions are obtained by performing a least-squares fit to the original density.

Stewart did not give computational prescription for the generation of these atoms, although he did show that they possess some useful properties. For example, the Stewart charges, obtained by integrating the Stewart atomic densities, reproduce the multipole moments of the molecule up to some order which depends on the number of symmetry-distinct atoms in the molecule. The nitrobenzene molecule (Fig. 1) has nine symmetry distinct atoms, and the Stewart charges (shown in Table 1) for this system are able to reproduce the charge and the first eight nonvanishing multipole moments. ${ }^{3}$ This is demonstrated in Table II which shows the moments obtained from the HF/6-31G $(d)$ density of the molecule, and those obtained from the Stewart and Mulliken partial charges placed on their respective nuclei. Both of these partial charge methods are well defined, and do not rely on fitting to electrostatic potential information evaluated on numerical grids. The Stewart charges are able to reproduce the moments up to hexadecapole order and consequently generate an accurate potential outside the molecular density, whereas the Mulliken charges fail to reproduce even the dipole of the system.

The fact that Stewart atoms, and their associated charges, are able to reproduce the most important multipole

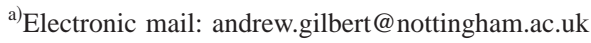

moments of a system is useful, however, this advantage is offset by the counterintuitive values obtained by the Stewart decomposition. The charges of the carbon atoms in nitrobenzene, for example, are large and alternate in sign around the aromatic ring, and the oxygen atoms support a very small positive charge. These results are not as odious as one might first think. There exists no quantum mechanical definition of an atom within a molecule and, therefore, any attempt to extract atomic identity is arbitrary. A good yardstick for assessing the quality of an atoms-within-molecules definition is its ability to reproduce observable quantities, such as the electrostatic potential. Figure 2 shows the potentials generated by the charges from Table I and the true HF/6-31G $(d)$ potential. The cross section is taken through the plane of the molecule and the contours are evenly spaced within the interval $[-0.01,0.01]$. The dotted line indicates the van der Waals surface. The Stewart charges provide a more faithful representation of the potential, particularly around the oxygen atoms, which have unexpected positive, but small, Stewart charges.

Although the Stewart charges of a molecule can be obtained relatively easily, ${ }^{3}$ the generation of the Stewart atoms themselves is more involved. The problem of extracting the Stewart atoms from a molecular density is still an important problem to consider, however, as the Stewart charges are not optimal in the same way that the Stewart atoms are. We have published a number of methods ${ }^{4-6}$ for the generation of approximate Stewart atoms from the electron density of an arbitrary molecule but the construction of the corresponding exact Stewart atoms proves to be a much more difficult problem. Nonetheless, in order to assess the accuracy of any proposed approximate technique, it is essential to have a method for generating exact atoms, even if that method is computationally expensive and applicable only to very small molecules. In this paper, we introduce such a method.

The Stewart density, $\tilde{\rho}$, can be obtained by minimizing the residual function 


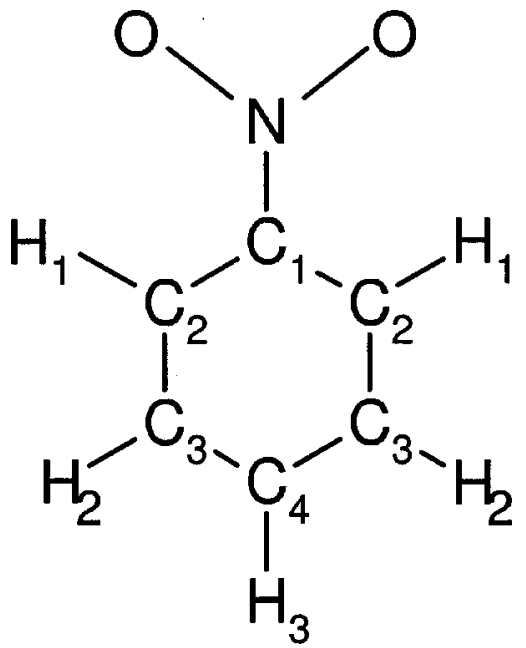

FIG. 1. The nitrobenzene molecule.

$Z_{\vartheta}=\iint\left[\rho\left(\mathbf{r}_{1}\right)-\tilde{\rho}\left(\mathbf{r}_{1}\right)\right] \vartheta\left(r_{12}\right)\left[\rho\left(\mathbf{r}_{2}\right)-\tilde{\rho}\left(\mathbf{r}_{2}\right)\right] d \mathbf{r}_{1} d \mathbf{r}_{2}$,

subject to the constraint that $\tilde{\rho}$ lies within the subspace spanned by a complete radial basis $\left\{\phi_{s}\right\}$ centered on each nucleus. The Stewart density may be written

$$
\tilde{\rho}(\mathbf{r})=\sum_{s j} c_{s j} \phi_{s}\left(r_{j}\right),
$$

where $r_{j}=\left|\mathbf{r}-\mathbf{A}_{j}\right|$ and $\mathbf{A}_{j}$ is the location of the $j$ th nucleus. The spherically symmetric Stewart atom $\sigma_{j}(r)$ is given by the linear combination of the basis functions on center $j$. In Eq. (1) $\vartheta$ is the fitting criterion which may be any positive definite operator, but is usually chosen to be one of $\delta\left(r_{12}\right)$, $r_{12}^{-1}$, or $-r_{12}$. These operators fit the density, electric field, and electric potential, respectively. ${ }^{7}$ It can be shown ${ }^{4}$ that the exact Stewart atoms are independent of this fitting operator, thus implying Stewart atoms are a simultaneous best-fit to the density, electric field and potential.

It has been shown that Stewart atoms can possess unexpected features such as regions of negative density and oscillations at large $r$. Given these pathologies, it is unsurprising that straightforward expansion of the Stewart atoms in a finite radial basis has met with limited success. In the following section we outline a method which avoids the use of such bases. Atomic units are used throughout.

\section{STEWART INTEGRAL EQUATIONS}

We define the spherical projection operator about center $i$

$$
S_{i}=\sum_{s}\left|\phi_{s}\left(r_{i}\right)\right\rangle\left\langle\phi_{s}\left(r_{i}\right)\right|,
$$

where, as before, the $\phi_{s}$ form a complete radial basis. The spherical projections of $\rho$ and $\tilde{\rho}$ are equivalent, and from the linearity of $S_{i}$ it follows that

$$
S_{i} \rho=S_{i} \tilde{\rho}=\sigma_{i}+\sum_{j \neq i} S_{i} \sigma_{j} .
$$

The spherical projection of the Stewart atom $\sigma_{j}$ about the center $i$ is given by

$$
\begin{aligned}
S_{i} \sigma_{j} & =\frac{1}{4 \pi} \int_{0}^{\pi} \int_{0}^{2 \pi} \sin (\theta) \sigma\left(\left|\mathbf{r}-\mathbf{R}_{i j}\right|\right) d \phi d \theta \\
& =\frac{1}{2} \int_{0}^{\pi} \sin (\theta) \sigma\left(\sqrt{r^{2}+R_{i j}^{2}-2 r R_{i j} \cos (\theta)}\right) d \theta,
\end{aligned}
$$

were $R_{i j}$ is the distance between centers $i$ and $j$. By making the substitution

$$
\begin{aligned}
& t=\sqrt{r^{2}+R_{i j}^{2}-2 r R_{i j} \cos (\theta)}, \\
& \frac{d t}{d \theta}=\frac{r R_{i j} \sin (\theta)}{t},
\end{aligned}
$$

the spherical projection can be written

$$
S_{i} \sigma_{j}=\frac{1}{2 R_{i j} r} \int_{r-R_{i j}}^{r+R_{i j}} t \sigma_{j}(t) d t,
$$

where we have assumed, without loss of generality, that the Stewart atoms are even functions. If we define Stewart functions $f_{i}$, local averages $\bar{f}_{i}$, and density projections $g_{i}$ as

$$
\begin{aligned}
& f_{i}(r)=r \sigma_{i}(r), \\
& \bar{f}_{i}(r ; R)=\frac{1}{2 R} \int_{r-R}^{r+R} f_{i}(t) d t, \\
& g_{i}(r)=r S_{i} \rho(\mathbf{r}),
\end{aligned}
$$

then Eq. (4) may be rewritten as coupled Fredholm equations of the second kind
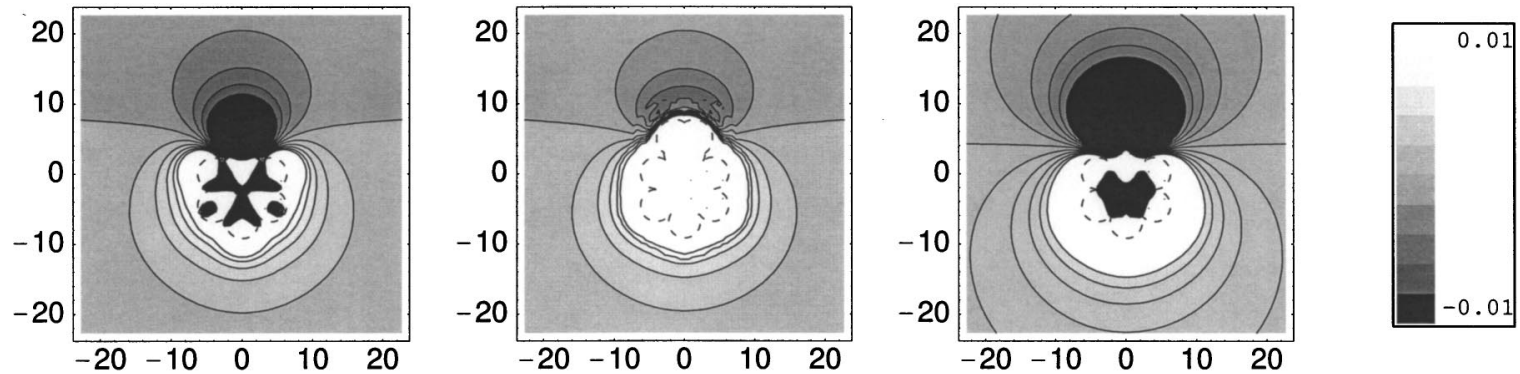

FIG. 2. Electrostatic potential for the HF/6-31G $(d)$ potential of nitrobenzene (center) and the corresponding potentials for the Stewart (left) and Mulliken (right) partial charges listed in Table I. 
TABLE I. Atomic partial charges for the HF/6-31G(d) density of nitrobenzene.

\begin{tabular}{lcccccccrr}
\hline \hline Atom & $\mathrm{C}_{1}$ & $\mathrm{C}_{2}$ & $\mathrm{C}_{3}$ & $\mathrm{C}_{4}$ & $\mathrm{H}_{1}$ & $\mathrm{H}_{2}$ & $\mathrm{H}_{3}$ & $\mathrm{~N}$ & $\mathrm{O}$ \\
\hline Stewart & 3.247 & -3.050 & 2.457 & -2.799 & 0.718 & -0.278 & 0.623 & -0.800 & 0.018 \\
Mulliken & 0.389 & -0.261 & -0.171 & -0.239 & 0.222 & 0.211 & 0.203 & 0.034 & -0.194 \\
\hline \hline
\end{tabular}

$$
f_{i}(r)+\sum_{j \neq i} \bar{f}_{j}\left(r ; R_{i j}\right)=g_{i}(r)
$$

Equation (13) can be written as a convolution

$$
\sum_{j} k_{i j} * f_{j}=g_{i}
$$

where the $k_{i j}$ are defined by

$$
k_{i j}(t)=\left\{\begin{array}{l}
1 / 2 R_{i j} \quad 0 \leqslant|t|<R_{i j} \\
0 \quad \text { otherwise }
\end{array}\right.
$$

and we have taken the limit $R \rightarrow 0$ to determine $h_{i i}(r)$ $=\delta(r)$. By taking the Fourier transforms of Eq. (14), solving for the Fourier transformed Stewart functions, and then back transforming, it is possible to show that the only physically reasonable solutions to Eq. (13) are given by

$$
f_{i}(r)=\sum_{j} \int h_{i j}(r-t) g_{j}(t) d t,
$$

where the $h_{i j}$ 's are the solution kernels. ${ }^{8}$ This may be expressed as the matrix equation

$$
\mathbf{f}=\mathbf{H} * \mathbf{g},
$$

where $\mathbf{f}$ is the vector of Stewart functions, $\mathbf{H}$ is the matrix of solution kernels, and $\mathbf{g}$ the vector of density projections. The solution kernels are given explicitly in terms of their inverse Fourier transforms

$$
h_{i j}=\mathrm{FT}^{-1}\left\{\frac{\hat{K}_{i j}}{|\mathbf{K}|}\right\},
$$

where $\mathbf{K}$ is the matrix of Fourier transforms of the $k_{i j}$ with entries $K_{i j}=j_{0}\left(R_{i j} r\right)$, and $\hat{K}_{i j}$ is the cofactor of the element $K_{i j}$.

An advantage of casting the problem in this form is that the integral equations provide a useful metric for determining the correctness of an approximate Stewart atom. Satisfying Eq. (13) is a necessary condition for exact Stewart atoms. Other tests based on matching the radial moments of the Stewart atoms are possible but the radial moments of the Stewart atoms depend on the multipole moments of the molecule and are, therefore, highly sensitive to small variations in the tail density.

TABLE II. Multipole moments for nitrobenzene (in atomic units) obtained from Stewart and Mulliken charges in Table I.

\begin{tabular}{lccc}
\hline \hline Multipole moment & Exact & Stewart & Mulliken \\
\hline$z$ & $-0.707210^{0}$ & $-0.707210^{0}$ & $-0.214010^{1}$ \\
$\frac{1}{2}\left(3 z^{2}-r^{2}\right)$ & $-0.108910^{1}$ & $-0.108910^{1}$ & $-0.576710^{1}$ \\
$\frac{\sqrt{3}}{2}\left(x^{2}-y^{2}\right)$ & $0.647310^{1}$ & $0.647210^{1}$ & $0.770310^{1}$ \\
$\frac{1}{2}\left(5 z^{2}-3 r^{2}\right) z$ & $-0.345610^{2}$ & $-0.345710^{2}$ & $-0.731910^{2}$ \\
$\frac{\sqrt{15}}{2}\left(x^{2}-y^{2}\right) z$ & $-0.305210^{2}$ & $-0.305210^{2}$ & $-0.503410^{2}$ \\
$\frac{1}{8}\left[8 z^{4}-24\left(x^{2}+y^{2}\right) z^{2}+3\left(x^{2}+y^{2}\right)^{2}\right]$ & $0.110910^{3}$ & $0.110910^{3}$ & $0.455810^{1}$ \\
$\frac{\sqrt{5}}{4}\left(x^{2}-y^{2}\right)\left(6 z^{2}-x^{2}-y^{2}\right)$ & $0.239210^{3}$ & $0.239210^{3}$ & $0.228310^{3}$ \\
$\frac{\sqrt{35}}{8}\left(x^{4}-6 x^{2} y^{2}+y^{4}\right)$ & $0.145410^{3}$ & $0.145410^{3}$ & $0.151410^{3}$ \\
$\frac{1}{4 \sqrt{2}}\left(15 r^{4}-70 r^{2} z^{2}+63 z^{4}\right) z$ & $-0.564010^{3}$ & $-0.191310^{4}$ & $-0.201110^{4}$ \\
$\frac{\sqrt{105}}{4}\left(x^{2}-y^{2}\right)\left(3 z^{2}-r^{2}\right) z$ & $-0.166810^{4}$ & $-0.231410^{4}$ & $-0.227110^{4}$ \\
$\frac{3 \sqrt{35}}{8}\left(x^{4}-6 x^{2} y^{2}+y^{4}\right) z$ & $-0.705910^{3}$ & $0.873610^{3}$ & $0.104110^{4}$ \\
\hline \hline
\end{tabular}




\section{DIATOMIC SOLUTION}

In the case of a diatomic molecule, Eqs. (13) can be cast into a dimensionless form via the scaling $x=r / R$, and taking the symmetry combinations

$$
\begin{aligned}
& f_{ \pm}=\left(f_{1} \pm f_{2}\right) / 2, \\
& g_{ \pm}=\left(g_{1} \pm g_{2}\right) / 2 .
\end{aligned}
$$

The equations then decouple to give

$$
f_{ \pm}(x)+\frac{1}{2} \int_{x-1}^{x+1} f_{ \pm}(t) d t=g_{ \pm}(x),
$$

and the solutions are given by the convolution

$$
f_{ \pm}=h_{ \pm} * g_{ \pm},
$$

where the solution kernels are given explicitly by

$$
h_{ \pm}(t)=\frac{1}{2 \pi} \int_{-\infty}^{\infty} \frac{\cos (k t)}{1 \pm j_{0}(k)} d k,
$$

and $j_{0}$ is the zeroth-order spherical Bessel function. These integrals do not exist in the Lebesgue sense, but their Cauchy principal values exist and these may be used to construct representations of the solution kernels.

By defining the functions

$$
\begin{aligned}
& \eta_{+}(k)=\frac{1}{1+j_{0}(k)}-1, \\
& \eta_{-}(k)=\frac{1}{1-j_{0}(k)}-\left(1+\frac{6}{k^{2}}\right),
\end{aligned}
$$

the problematic parts of the integrands in (22) can be isolated and their Fourier transforms evaluated analytically, whence

$$
\begin{aligned}
& h_{+}(t)=\delta(t)+\frac{1}{2 \pi} \int_{-\infty}^{\infty} \eta_{+}(k) \cos (k t) d k, \\
& h_{-}(t)=\delta(t)-3|t|+\frac{1}{2 \pi} \int_{-\infty}^{\infty} \eta_{-}(k) \cos (k t) d k .
\end{aligned}
$$

Although we have been unable to evaluate the remaining integrals analytically, they are more amenable to approximation than those in Eq. (22); the functions $\eta_{ \pm}(k)$ decay as $1 / k$, and are free from singularities. Let $\left\{\varphi_{i}\right\}$ be a complete, even basis in real space. The Fourier transforms of these functions generate a corresponding basis $\left\{\Phi_{i}\right\}$ in Fourier space. If $c_{i}^{ \pm}$are the expansion coefficients of $\eta_{ \pm}$in the Fourier basis, then the limiting value theorem ${ }^{9}$ allows us to write

$$
\begin{aligned}
& h_{+}(t)=\delta(t)+\sum_{i} c_{i}^{+} \varphi_{i}(t), \\
& h_{-}(t)=\delta(t)-3|t|+\sum_{i} c_{i}^{-} \varphi_{i}(t) .
\end{aligned}
$$

Although many possibilities exist for the choice of basis, we confine our attention to those for which the Fourier transforms exist, and for which the convolution integrals in (21) can be performed analytically. Preliminary investigations

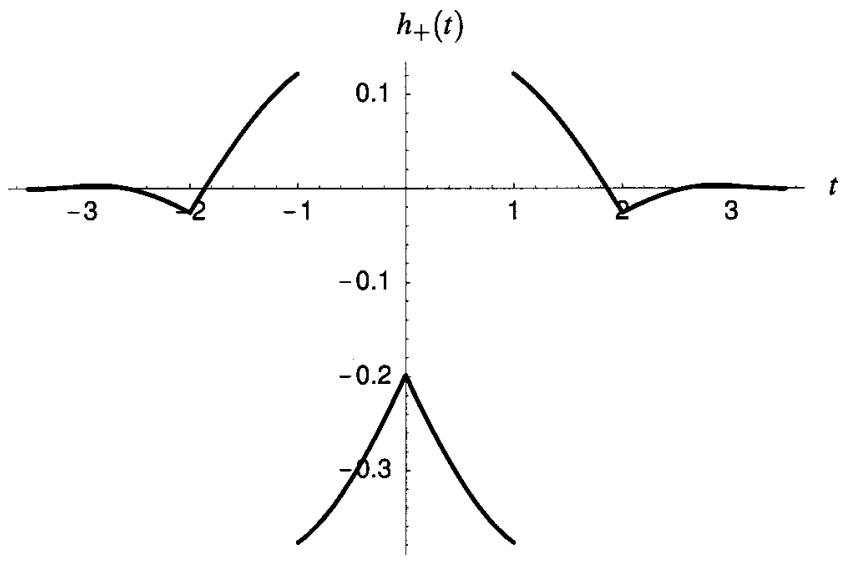

FIG. 3. The $h_{+}(t)$ solution kernel.

lead us to use piecewise polynomials. For convenience we introduce the unit step function $U$, and re-index the $\varphi_{i}$ basis functions as follows:

$$
\begin{aligned}
& U(t)= \begin{cases}1 & 0 \leqslant t<1 \\
0 & \text { otherwise, }\end{cases} \\
& \varphi_{\mu \nu}(t)=|t|^{\nu}[U(|t|-\mu)+U(|t|+\mu)] \quad \mu, \nu \in \mathbb{Z} .
\end{aligned}
$$

The Fourier transforms of these functions are straightforward to evaluate, and yield an oscillatory basis in Fourier space that was used to fit the $\eta$ functions. The fitting was carried out using the MATHEMATICA package. ${ }^{10}$ The $\eta$ functions were truncated for $|t|>9$, at which point they had decayed to less than $5 \times 10^{-9}$, and $\nu_{\max }$ was chosen to be 6 . The maximum difference between the $\nu_{\max }=6$ and $\nu_{\max }=7$ approximations on the interval $[-9,9]$ is $3 \times 10^{-8}$. The resulting kernels (without the delta functions) are shown in Figs. 3 and 4.

Both kernels have a delta function at the origin and are discontinuous at \pm 1 . Derivative discontinuities exist at integer values, but these discontinuities occur in higher-order derivatives as $t$ increases. As a check of the accuracy of the approximate kernels, their low-order moments were computed and compared to the correct values. The zeroth-order moment of the approximation to $h_{+}$was correct to six significant figures. The reproduction of this moment of $h_{+}$is sufficient to ensure charge conservation. For a homonuclear

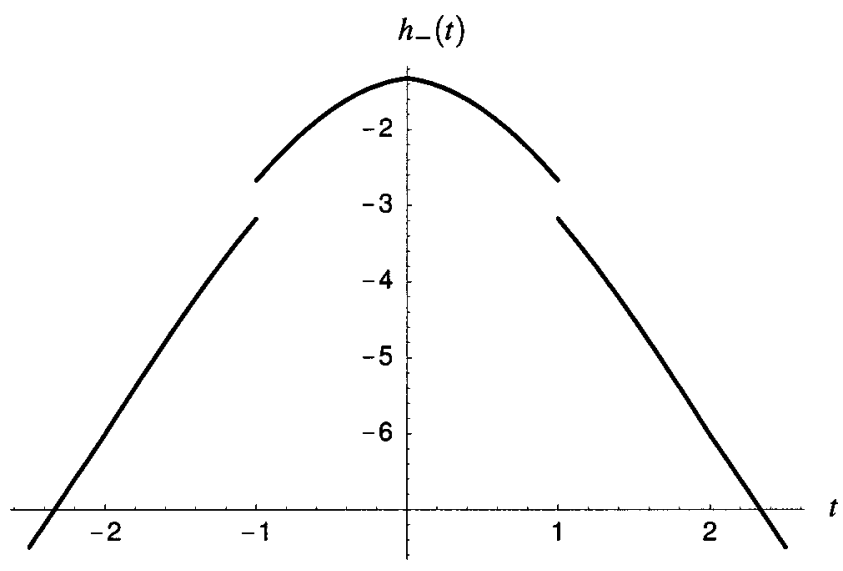

FIG. 4. The $h_{-}(t)$ solution kernel. 
diatomic, $g_{-}$is zero and the $h_{+}$kernel is sufficient to determine the Stewart atoms. In the case of a heteronuclear diatomic molecule, both solution kernels are required.

\section{COMPUTING THE CONVOLUTION INTEGRALS}

The molecular density is usually expressed in terms of the density matrix $\mathbf{P}$ and products of orbital basis functions $\chi$,

$$
\rho(\mathbf{r})=\sum_{\mu \nu} P_{\mu \nu} \chi_{\mu}(\mathbf{r}) \chi_{\nu}(\mathbf{r}) .
$$

For computational reasons the $\chi_{\mu}$ are usually chosen to be Gaussian functions. Convolution is a linear operation so we need only consider the effect of the solution kernels on a single basis function pair, $\chi_{\mu} \chi_{\nu}$. If the two basis functions are of $s$-type and are centered on $\mathbf{A}$ and $\mathbf{B}$, respectively, then the spherical average about a third center $\mathbf{C}$ is given by

$$
\begin{aligned}
g_{s s}(r) & =1 /(4 \pi) \int e^{-\alpha|\mathbf{r}-(\mathbf{A}-\mathbf{C})|^{2}} e^{-\beta|\mathbf{r}-(\mathbf{B}-\mathbf{C})|^{2}} d \Omega \\
& =\frac{\sinh (2 \zeta Q r)}{2 \zeta Q} e^{-\zeta\left(r^{2}+Q^{2}\right)} e^{\left(-\alpha \beta|\mathbf{A}-\mathbf{B}|^{2} /(\alpha+\beta)\right)},
\end{aligned}
$$

where

$$
\mathbf{Q}=\frac{\alpha \mathbf{A}+\beta \mathbf{B}}{\alpha+\beta}-\mathbf{C},
$$

$\zeta=\alpha+\beta$ and $\Omega$ is the angular part of $\mathbf{r}$. Integrals involving basis functions of higher angular momentum can be found by differentiating $g_{s s}$ with respect to the centers $\mathbf{A}$ and $\mathbf{B}$. Expanding the kernels, $h_{ \pm}(r-t)$, using the binomial theorem results in integrals of the form

$$
\int_{a}^{b} t^{n} g(t) d t
$$

which are tedious, but straightforward, to evaluate for each value of $n$.

\section{HETERONUCLEAR DIATOMIC EXAMPLE}

We have implemented the above convolution scheme in a development version of the QCHEM package ${ }^{11}$ and, as an example of its use, applied it to the hydrogen fluoride molecule, FH. The spherical projections, $g(r)$, were obtained from Hartree-Fock densities computed using the 6-31G basis set. Radial distribution functions of the Stewart atoms are shown in Figs. 5 and 6.

In each case the error in the integrated density was not more than one part in $10^{6}$, which accords with the reproduction of the zeroth moment for $h_{+}$to six significant figures. For small $r$ the Stewart atoms are almost identical to the spherical average of the isolated atoms, but for larger $r$ the effects of the other atom in the system are evident. This is particularly noticeable for the hydrogen atom where contributions to the density are spread almost equally between the nucleus and a shell with a similar radius as the bond length.

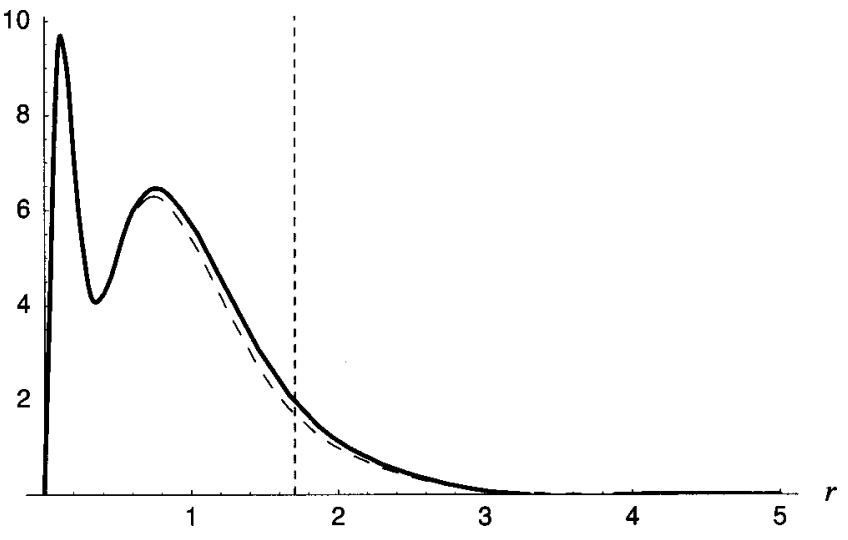

FIG. 5. $4 \pi r^{2} \sigma(r)$ for the fluorine Stewart atom in the FH molecule using $\mathrm{HF} / 6-31 \mathrm{G}(d)$ (in bold). The dashed curve shows $4 \pi r^{2} \rho(r)$ for the atomic density, and the dotted line the bond length. $\mathrm{R}(\mathrm{H}-\mathrm{F})=1.703 \mathrm{bohr}$.

The complex shape of this Stewart atom gives an indication of the flexibility required in a basis set, if one were used to expand the Stewart atom.

Figure 7 shows the difference between the true HF/6$31 \mathrm{G}$ electrostatic potential (excluding the nuclei) for $\mathrm{FH}$, and the corresponding potential of the Stewart density. The molecule is oriented in the vertical direction with the center of nuclear charge at the origin. The dotted line represents the van der Waals surface. The Stewart potential is a faithful representation of the true potential even inside the charge density where an atomic charge or multipole expansion would break down. The Stewart model is exact at the nuclei, which is a consequence of the fitting procedure. In the asymptotic regions the potential is dominated by the strong dipole of this system $\left(0.905 e a_{0}\right)$. This dipole is exactly reproduced by the Stewart density, as is the total (zero) charge. In this small system the single nonzero component of the quadrupole tensor $\left(\left(3 z^{2}-r^{2}\right) / 2\right)$ is not reproduced correctly - the true value being $1.430 e a_{0}^{2}$, whereas that of the Stewart density is $1.260 e a_{0}^{2}$. The approximation is worst in the bonding region of the system where the buildup of density on one side of each atom makes it difficult for the Stewart model to capture the exact behavior. Nevertheless, the $400 m E_{h}$ difference represents a relative error of less than $4 \%$ at this point. The relative error at any point outside the van der Waals radius is less than $1.5 \%$. The accuracy of the

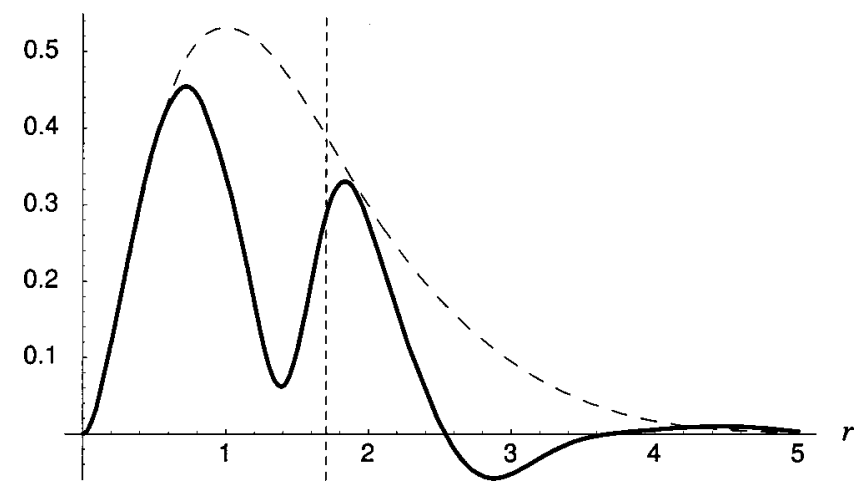

FIG. 6. $4 \pi r^{2} \sigma(r)$ for the hydrogen Stewart atom in the FH molecule using $\mathrm{HF} / 6-31 \mathrm{G}(d)$ (in bold). The dashed curve shows $4 \pi r^{2} \rho(r)$ for the atomic density, and the dotted line the bond length. $\mathrm{R}(\mathrm{H}-\mathrm{F})=1.703$ bohr. 


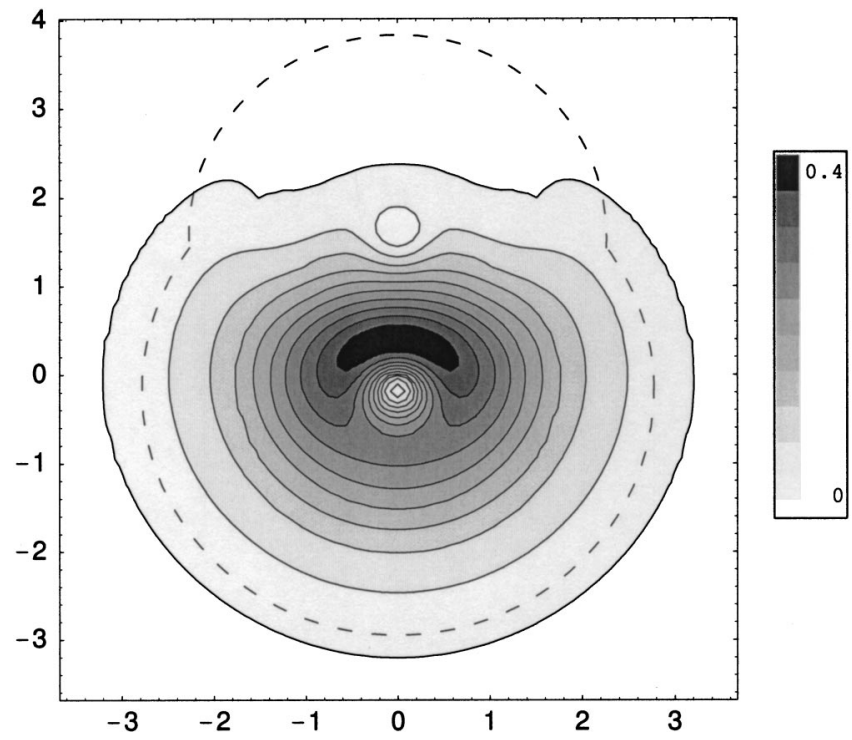

FIG. 7. Cross-section of the difference between the electrostatic potentials (excluding the nuclei) of the HF/6-31G density for hydrogen fluoride, and the potential for the corresponding Stewart density. The dashed line indicates the van der Waals surface. The cross-section is taken along the bond axis. The fluorine atom is located at $-0.170 a_{0}$ and the hydrogen at $1.53 a_{0}$.

Stewart potential derives from the fact that the Stewart density provides a global best-fit to not only the density, but also the potential of a system.

\section{POLYATOMIC MOLECULES}

The simplest triatomic system that can be considered is an equilateral $\mathrm{A}_{3}$ molecule, for example $\mathrm{H}_{3}^{+}$. The three Stewart atoms are equivalent and upon normalization of the bond length the integral equations (13) become

$$
f(x)+2 \bar{f}(x ; 1)=g(x) .
$$

The symmetry-adapted solution kernel is given by

$$
h_{3}(t)=\frac{1}{2 \pi} \int_{-\infty}^{\infty} \frac{\cos (k t)}{1+2 j_{0}(k)} d k,
$$

and is plotted in Fig. 8. This solution kernel is qualitatively similar to the symmetry adapted kernel for the homonuclear diatomic, Fig. 3. Both exhibit discontinuities at \pm 1 (the nor-

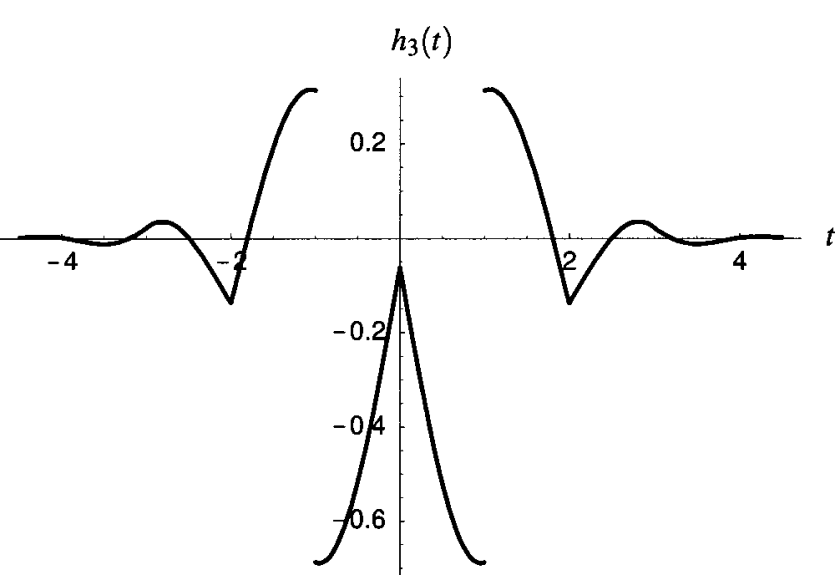

FIG. 8. The $h_{3}(t)$ solution kernel.

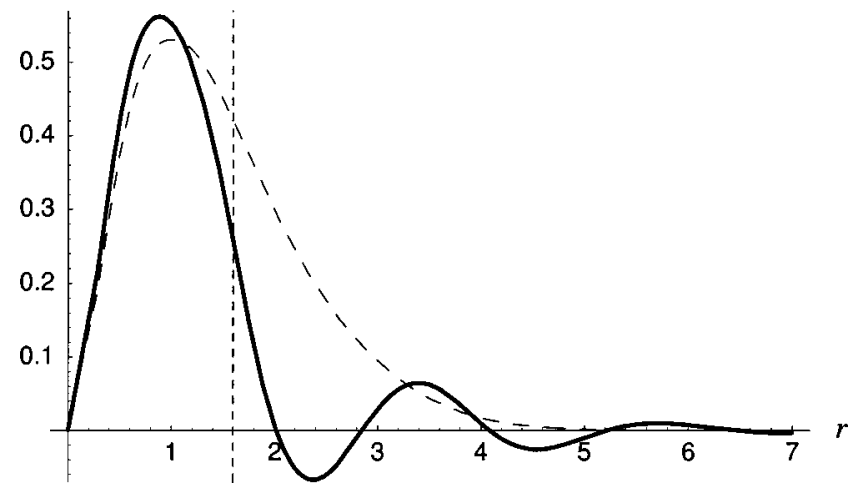

FIG. 9. $4 \pi r^{2} \sigma(r)$ for the hydrogen Stewart atom in the $\mathrm{H}_{3}^{+}$ion using HF/6-31G (in bold). The dashed curve shows $4 \pi r^{2} \rho(r)$ for the atomic density, and the dotted line the bond length. $\mathrm{R}(\mathrm{H}-\mathrm{H})=1.596 \mathrm{bohr}$.

malized bond length), derivative discontinuities at larger integer values and decay rapidly in an oscillatory manor. The Stewart atom in $\mathrm{H}_{3}^{+}$is shown in Fig. 9 and, like the hydrogen Stewart atom in the FH molecule, it is oscillatory.

The negative regions of the Stewart atom in this system occur beyond the bond length, and reflect the aspherical environment about each nucleus. There is a build up of density between the nuclei in the bonding regions, and a corresponding depletion of density outside the nuclear framework. The spherical constraint placed on the Stewart atoms makes it impossible for each atom to model this distortion locally, and therefore, they must enlist the help of the other atoms in the system to remove density from the nonbonding side of the nucleus. This interpretation is supported by the shape of the helium Stewart atom in the hypothetical $\mathrm{He}_{3}$ system, shown in Fig. 10. This system is not bound, and rather than a depletion of electron density on the nonbonding side of the nuclei, there is, in fact, a build up of density due to the Pauli repulsion pushing electron density outside the inter-nuclear region.

The general triatomic is more problematic as it contains three different internuclear distances. Normalization is not possible, and the kernels depend parametrically on the geometry of the system. One possible way forward is to generate the kernels dynamically for a given geometry, however, the

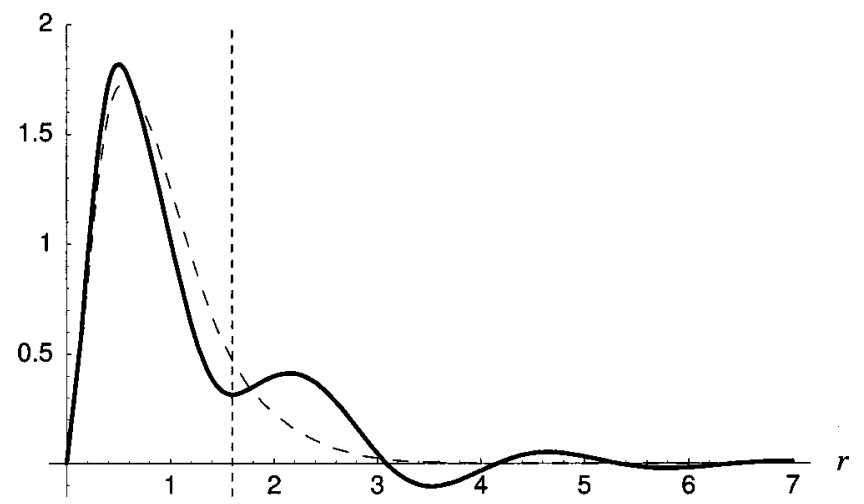

FIG. 10. $4 \pi r^{2} \sigma(r)$ for the helium Stewart atom in the hypothetical $\mathrm{He}_{3}$ molecule using HF/6-31G (in bold). The dashed curve shows $4 \pi r^{2} \sigma(r)$ for the atomic density, and the dotted line the bond length. $\mathrm{R}(\mathrm{He}-\mathrm{He})=1.596$ bohr. 
cost of this approach scales very poorly with the number of centers due to the determinant term in the kernel definition (18).

\section{CONCLUSIONS}

We have presented an approach for generating accurate Stewart atoms from molecular densities based on solution kernels of the Stewart integral equations. Explicit representations of the diatomic kernels have been found, and these can be used to determine the Stewart atoms in any diatomic system to high accuracy. In principle the approach can be extended to larger systems, however, the solution kernels become dependent on the geometry of the molecule and an efficient scheme for generating the kernels on-the-fly must first be determined.

\section{ACKNOWLEDGMENT}

ATBG is grateful for funding and support from QCHEM Inc.

${ }^{1}$ R. F. Stewart, E. R. Davidson, and W. J. Simpson, J. Chem. Phys. 42, 3175 (1965).

${ }^{2}$ R. F. Stewart, Isr. J. Chem. 16, 124 (1977).

${ }^{3}$ A. C. Simmonett, A. T. B. Gilbert, and P. M. W. Gill (in preparation).

${ }^{4}$ P. M. W. Gill, J. Phys. Chem. 100, 15421 (1996).

${ }^{5}$ A. M. Lee and P. M. W. Gill, Chem. Phys. Lett. 286, 226 (1998).

${ }^{6}$ A. T. B. Gilbert, A. M. Lee, and P. M. W. Gill, J. Mol. Struct.: THEOCHEM 500, 363 (2000).

${ }^{7}$ P. M. W. Gill, B. G. Johnson, J. A. Pople, and S. W. Taylor, J. Chem. Phys. 96, 7178 (1992)

${ }^{8}$ D. Porter and D. S. G. Stirling, Integral Equations, a Practical Treatment from Spectral Theory to Applications (Cambridge University Press, Cambridge, 1996), p. 330.

${ }^{9}$ I. N. Bronshtein and K. A. Semendyayev, Handbook of Mathematics (Springer-Verlag, Berlin, 1997), p. 583.

${ }^{10}$ Wolfram Research Inc., MATHEMATiCA 4.0, 1999.

${ }^{11}$ J. Kong et al., J. Comput. Chem. 21, 1532 (2000). 